PROCEEDINGS OF THE

AMERICAN MATHEMATICAL SOCIETY

Volume 125, Number 6, June 1997, Pages 1745-1749

S 0002-9939(97)03750-7

\title{
THE BEURLING-MALLIAVIN DENSITY OF A RANDOM SEQUENCE
}

\author{
KRISTIAN SEIP AND ALEXANDER M. ULANOVSKII
}

(Communicated by J. Marshall Ash)

\begin{abstract}
A formula is given for the completeness radius of a random exponential system $\left\{t^{l} e^{i \xi_{n} t}\right\}_{l=0, n \in \mathbb{Z}}^{p_{n}-1}$ in terms of the probability measures of $\xi_{n}$.
\end{abstract}

The purpose of this note is to formulate a probabilistic version of the celebrated Beurling-Malliavin theorem on the completeness radius of a sequence of complex exponentials [1]. First, to state the Beurling-Malliavin theorem, let $\Lambda=\left\{\lambda_{n}\right\}_{n \in \mathbb{Z}}$ be a sequence of real numbers and $\left\{p_{n}\right\}_{n}$ an associated sequence of positive integers, so that $p_{n}$ denotes the multiplicity of $\lambda_{n}$ in $\Lambda$. The completeness radius $R(\Lambda)$ of $\mathcal{E}(\Lambda)=\left\{t^{l} e^{i \lambda_{n} t}\right\}_{l=0, n \in \mathbb{Z}}^{p_{n}-1}$ is defined as the supremum over all $R \geq 0$ such that $\mathcal{E}(\Lambda)$ is a complete sequence in $L^{2}(-R, R)$. The Beurling-Malliavin theorem states that $R(\Lambda)=\pi D(\Lambda)$, where $D(\Lambda)$ is the Beurling-Malliavin density of $\Lambda$. Following [3], we define this density as follows. A system of intervals $\left\{\left(a_{n}, b_{n}\right)\right\}_{n \in \mathbb{N}}, a_{n}<b_{n}$, is substantial if either $0<a_{1}<b_{1} \leq a_{2}<b_{2} \ldots$ and $\sum_{n \in \mathbb{N}} a_{n}^{-2}\left(b_{n}-a_{n}\right)^{2}=\infty$, or $0>b_{1}>a_{1} \geq b_{2}>a_{2} \ldots$ and $\sum_{n \in \mathbb{N}}\left|b_{n}\right|^{-2}\left(b_{n}-a_{n}\right)^{2}=\infty$. If $\Lambda$ has a finite accumulation point, then $D(\Lambda)=\infty$. Otherwise, $D(\Lambda)$ is defined as the supremum over all $R \geq 0$ for which there exists a substantial sequence of intervals $\left\{\left(a_{n}, b_{n}\right)\right\}_{n \in \mathbb{N}}$ such that the number of elements of $\Lambda$ on each interval $\left(a_{n}, b_{n}\right)$ is greater than or equal to $R\left(b_{n}-a_{n}\right)$.

If $\Xi=\left\{\xi_{n}\right\}_{n \in \mathbb{Z}}$ is a sequence of independent random variables, we may study completeness problems for the random exponential system $\left\{t^{l} \exp \left(i \xi_{n} t\right)\right\}_{l=0, n \in \mathbb{Z}}^{p_{n}-1}$, where, as above, $p_{n}<\infty$ denotes the multiplicity of the point $\xi_{n}$. Recently, this approach has proved to be fruitful and has led to new insight into classical problems [2], [4]. While [2], [4] deal with random perturbations of a fixed sequence, we now address the problem of computing the Beurling-Malliavin density of an arbitrary random sequence.

To solve this problem, we need the Beurling-Malliavin density of a Borel (not necessarily locally finite) measure. If $\mu$ is not locally finite, we set $d(\mu)=\infty$. Otherwise, the Beurling-Malliavin density $d(\mu)$ is defined as the supremum over all $R \geq 0$ for which there exists a substantial sequence $\left\{\left(a_{n}, b_{n}\right)\right\}_{n \in \mathbb{N}}$ such that $\mu\left(\left(a_{n}, b_{n}\right)\right) \geq$ $R\left(b_{n}-a_{n}\right)$ for every $n \in \mathbb{N}$. Observe that to any sequence $\Lambda=\left\{\lambda_{n}\right\}_{n \in \mathbb{Z}}$ one can associate a measure $\nu=\sum_{n \in \mathbb{Z}} \delta_{\lambda_{n}}$, where $\delta_{x}$ is a unit measure concentrated at

Received by the editors September 8, 1995 and, in revised form, December 11, 1995.

1991 Mathematics Subject Classification. Primary 42A61, 42C30.

Research supported by NATO linkage grant LG 930329.

(C)1997 American Mathematical Society 
$x$. We have $D(\Lambda)=d(\nu)$, in which sense the density $d$ is a generalization of the density $D$.

We shall prove:

Theorem. Let $\Xi=\left\{\xi_{n}\right\}_{n \in \mathbb{Z}}$ be a sequence of independent random variables and $\nu_{\Xi}=\sum_{n \in \mathbb{Z}} \mu_{n}$, where $\mu_{n}((-\infty, x))=\mathbf{P}\left(\xi_{n}<x\right)$ are the probability measures of $\xi_{n}$. Then, with probability one, $D(\Xi)=d\left(\nu_{\Xi}\right)$, i.e., $R(\Xi)=\pi d\left(\nu_{\Xi}\right)$.

Let us denote by $n_{\Lambda}(I)$ the number of elements from a sequence $\Lambda$ in an interval $I$. For the proof of the Theorem, we shall need two lemmas.

Lemma 1. Suppose $\Xi=\left\{\xi_{n}\right\}_{n \in \mathbb{Z}}$ is a sequence of independent random variables such that the measure $\nu_{\Xi}$ defined in the Theorem is locally finite. Then the inequality

$$
\mathbf{P}\left(\left|n_{\Xi}(I)-\nu_{\Xi}(I)\right|>\alpha\right) \leq \frac{(2 n) ! e \max \left\{1,\left(\nu_{\Xi}(I)\right)^{n}\right\}}{\alpha^{2 n}}
$$

holds for all $\alpha>0$ and $n \in \mathbb{N}$.

Proof. Denote by $\chi_{I}(x)$ the characteristic function of $I$. Then $n_{\Xi}(I)=\sum_{n \in \mathbb{Z}} \chi_{I}\left(\xi_{n}\right)$. Observe that

$$
\mathbf{E} e^{t \chi_{I}\left(\xi_{n}\right)}=\mu_{n}(\mathbb{R} \backslash I)+e^{t} \mu_{n}(I),
$$

where $\mu_{n}((-\infty, x))=\mathbf{P}\left(\xi_{n}<x\right)$ and $\mathbf{E}$ denotes mathematical expectation. Since the $\xi_{n}$ are independent, we obtain

$$
\begin{gathered}
\mathbf{E} e^{t(n \Xi(I)-\nu \Xi(I))}=e^{-t \nu \Xi(I)} \prod_{n \in \mathbb{Z}} \mathbf{E} e^{t \chi_{I}\left(\xi_{n}\right)}=e^{-t \nu \Xi(I)} \prod_{n \in \mathbb{Z}}\left(1+\left(e^{t}-1\right) \mu_{n}(I)\right) \\
\leq e^{-t \nu_{\Xi}(I)} \prod_{n \in \mathbb{Z}} e^{\left(e^{t}-1\right) \mu_{n}(I)}=e^{\left(e^{t}-t-1\right) \nu_{\Xi}(I)} .
\end{gathered}
$$

Thus, since

$$
\frac{2 t^{2 n}}{(2 n) !}\left(n_{\Xi}(I)-\nu_{\Xi}(I)\right)^{2 n}<e^{t(n \Xi(I)-\nu \Xi(I))}+e^{-t(n \Xi(I)-\nu \Xi(I))}
$$

we have

$$
\begin{aligned}
\mathbf{E}\left(n_{\Xi}(I)-\nu_{\Xi}(I)\right)^{2 n} & \leq \frac{(2 n) !}{2 t^{2 n}} \mathbf{E}\left(\exp t\left(n_{\Xi}(I)-\nu_{\Xi}(I)\right)+\exp \left\{-t\left(n_{\Xi}(I)-\nu_{\Xi}(I)\right)\right\}\right) \\
& \leq \frac{(2 n) !}{2 t^{2 n}}\left(e^{\left(e^{t}-t-1\right) \nu_{\Xi}(I)}+e^{\left(e^{-t}+t-1\right) \nu_{\Xi}(I)}\right) .
\end{aligned}
$$

We put $t=\min \left\{1,\left(\nu_{\Xi}(I)\right)^{-1 / 2}\right\}$ and observe that $e^{t}-t-1<t^{2}$ for all $|t| \leq 1$. Hence

$$
\mathbf{E}\left(n_{\Xi}(I)-\nu_{\Xi}(I)\right)^{2 n} \leq(2 n) ! e \max \left\{1,\left(\nu_{\Xi}(I)\right)^{n}\right\} .
$$

Now (1) follows from Chebyshev's inequality

$$
\mathbf{P}(|\eta|>\alpha)<\frac{\mathbf{E} \eta^{2 n}}{\alpha^{2 n}}
$$

which holds for every random variable $\eta$ with $\alpha>0$ and $n \in \mathbb{N}$. 
Lemma 2. Let $\left\{\left(a_{n}, b_{n}\right)\right\}_{n \in \mathbb{N}}$ be a substantial sequence of intervals. For any $N>$ 1 and $\epsilon>0$ there exists a set $S \subset \mathbb{N}$ such that the sequence $\left\{\left(a_{n}, b_{n}\right)\right\}_{n \in S}$ is substantial, $b_{n}-a_{n}>N$ for all $n \in S$ and

$$
\sum_{n \in S}\left(b_{n}-a_{n}\right)^{-3}<\epsilon .
$$

Proof. Without loss of generality, we assume that our sequence of intervals is such that $0<a_{1}<b_{1} \leq a_{2} \ldots$ and $\sum_{n \in \mathbb{N}} a_{n}^{-2}\left(b_{n}-a_{n}\right)^{2}=\infty$. Denote by $Q_{N}$ the set of all $n \in \mathbb{N}$ such that $b_{n}-a_{n} \leq N$. We have

$$
\begin{gathered}
\sum_{n \in Q_{N}}\left(\frac{b_{n}-a_{n}}{a_{n}}\right)^{2} \leq N \frac{a_{1}+N}{a_{1}} \sum_{n \in Q_{N}} \frac{b_{n}-a_{n}}{a_{n} b_{n}}=N \frac{a_{1}+N}{a_{1}} \sum_{n \in Q_{N}}\left(\frac{1}{a_{n}}-\frac{1}{b_{n}}\right) \\
\leq N \frac{a_{1}+N}{a_{1}^{2}}<\infty .
\end{gathered}
$$

It follows that the sequence $\left\{\left(a_{n}, b_{n}\right)\right\}_{n \in \mathbb{N} \backslash Q_{N}}$ is substantial. Denote by $Q$ the set of $n \in \mathbb{N}$ such that $b_{n} \leq a_{n}+a_{n} n^{-5 / 8}$. Since

$$
\sum_{n \in \mathbb{N}}\left(\frac{b_{n}-a_{n}}{a_{n}}\right)^{2} \leq \sum_{n \in \mathbb{N}} n^{-5 / 4}<\infty,
$$

we conclude that the sequence $\left\{\left(a_{n}, b_{n}\right)\right\}_{n \in \mathbb{N} \backslash\left(Q \cup Q_{N}\right)}$ is substantial. Thus we may assume that $b_{n}-a_{n}>N$ and $b_{n}>a_{n}+a_{n} n^{-5 / 8}$ for all $n \in \mathbb{N}$. The first of these inequalities yields $a_{n}>N(n-1)$, and so

$$
\sum_{n \in \mathbb{N}}\left(b_{n}-a_{n}\right)^{-3} \leq\left(b_{1}-a_{1}\right)^{-3}+\sum_{n \geq 2}\left(N(n-1) n^{-5 / 8}\right)^{-3}<\infty .
$$

Hence, for $n_{\epsilon}$ large enough we have $\sum_{n>n_{\epsilon}}\left(b_{n}-a_{n}\right)^{-3}<\epsilon$, which completes the proof of the lemma.

We are now in position to prove the Theorem. Let us denote by $\Omega$ a probability space on which all the $\xi_{n}$ are defined.

(i) Suppose first that the measure $\nu_{\Xi}$ is not locally finite, i.e. $\nu_{\Xi}(I)=\infty$ for some interval $I$. It follows that $\mathbf{E} n_{\Xi}(I)=\infty$. By the Borel-Cantelli Lemma, with probability one the number of $\xi_{n}$ which belong to $I$ is $\infty$, so we conclude that with probability one $R(\Xi)=\infty$.

(ii) Suppose that $\nu_{\Xi}$ is locally finite and $d\left(\nu_{\Xi}\right)>0$. Let us prove that

$$
\mathbf{P}\left(R(\Xi)<d\left(\nu_{\Xi}\right)\right)=0 .
$$

Fix an arbitrary $\rho, 0<\rho<\frac{1}{2} d\left(\nu_{\Xi}\right)$, and take a substantial sequence of intervals $\left\{\left(a_{n}, b_{n}\right)\right\}_{n \in \mathbb{Z}}$ such that

$$
\left(b_{n}-a_{n}\right)^{-1} \nu_{\Xi}\left(\left(a_{n}, b_{n}\right)\right) \geq d\left(\nu_{\Xi}\right)-\rho
$$

for all $n$. By Lemma 2, we may assume that

$$
\sum_{n \in \mathbb{N}}\left(b_{n}-a_{n}\right)^{-3}<\frac{\rho^{7}}{6 ! e}\left(d\left(\nu_{\Xi}\right)-\rho\right)^{3}
$$


and $\nu_{\Xi}\left(\left(a_{n}, b_{n}\right)\right)>1$ for all $n$. It follows from Lemma 1 that

$$
\begin{gathered}
\mathbf{P}\left(n_{\Xi}\left(a_{n}, b_{n}\right) \geq(1-\rho) \nu_{\Xi}\left(\left(a_{n}, b_{n}\right)\right) \text { for all } n\right) \\
\geq \mathbf{P}\left(\left|n_{\Xi}\left(a_{n}, b_{n}\right)-\nu_{\Xi}\left(\left(a_{n}, b_{n}\right)\right)\right| \leq \rho \nu_{\Xi}\left(\left(a_{n}, b_{n}\right)\right) \text { for all } n\right) \\
\geq 1-\sum_{n \in \mathbb{N}} \mathbf{P}\left(\left|n_{\Xi}\left(a_{n}, b_{n}\right)-\nu_{\Xi}\left(\left(a_{n}, b_{n}\right)\right)\right|>\rho \nu_{\Xi}\left(\left(a_{n}, b_{n}\right)\right)\right) \\
>1-6 ! e \rho^{-6}\left(d\left(\nu_{\Xi}\right)-\rho\right)^{-3} \sum_{n \in \mathbb{N}}\left(b_{n}-a_{n}\right)^{-3}>1-\rho .
\end{gathered}
$$

Hence, with probability at least $1-\rho$, we have $\left(\nu_{\Xi}\left(\left(a_{n}, b_{n}\right)\right)\right)^{-1} n_{\Xi}\left(a_{n}, b_{n}\right) \geq 1-\rho$ for all $n$. Thus, with probability at least $1-\rho$, we obtain $R(\Xi)=\infty$ if $d\left(\nu_{\Xi}\right)=\infty$ and $R(\Xi) \geq(1-\rho)\left(d\left(\nu_{\Xi}\right)-\rho\right)$ if $d\left(\nu_{\Xi}\right)<\infty$. Letting $\rho \rightarrow 0$, we obtain (2).

(iii) It remains to be shown that if $\nu_{\Xi}$ is locally finite and $d\left(\nu_{\Xi}\right)<\infty$, then

$$
\mathbf{P}\left(R(\Xi)>d\left(\nu_{\Xi}\right)\right)=0 .
$$

Clearly, we may assume, without loss of generality, that $\nu_{\Xi}$ is supported on $\mathbb{R}^{+}$.

By Lemma 2, it is enough to consider substantial sequences for which $a_{n}>n$. Since then

$$
\sum_{b_{n} \leq a_{n}+a_{n}^{1 / 4}}\left(\frac{b_{n}-a_{n}}{a_{n}}\right)^{2}<\sum_{n \geq 1} n^{-3 / 2}<\infty,
$$

the intervals for which $b_{n}>a_{n}+a_{n}^{1 / 4}$ constitute a substantial sequence. By the definition of $d\left(\nu_{\Xi}\right)$, the inequality $\nu_{\Xi}\left(\left(a_{n}, b_{n}\right)\right) \leq(3 / 2) d\left(\nu_{\Xi}\right)\left(b_{n}-a_{n}\right)$ holds for infinitely many such $n$. Thus, if for some $\omega \in \Omega$ and $\epsilon>0$, we have

$$
R(\Xi(\omega)) \geq d\left(\nu_{\Xi}\right)+2 \epsilon
$$

then the inequalities

$$
n_{\Xi(\omega)}(k, k+m)>\nu_{\Xi}((k, k+m))+\epsilon m, \nu_{\Xi}((k, k+m)) \leq 2 d\left(\nu_{\Xi}\right) m
$$

hold for infinitely many $k=2,3, \ldots$ and some $m>k^{1 / 4}$. It follows that for $\epsilon>0$

$$
\begin{aligned}
&\{\omega:\left.R(\Xi) \geq d\left(\nu_{\Xi}\right)+2 \epsilon\right\} \subset \\
& \bigcap_{j>1} \bigcup_{k>j} \bigcup_{m>k^{1 / 4}}\left\{\omega:\left|n_{\Xi}(k, k+m)-\nu_{\Xi}((k, k+m))\right|>\epsilon m,\right. \\
&\left.\nu_{\Xi}((k, k+m)) \leq 2 d\left(\nu_{\Xi}\right) m\right\} .
\end{aligned}
$$

Thus, choosing $n=6$ in (1), we obtain

$$
\mathbf{P}\left(R(\Xi) \geq d\left(\nu_{\Xi}\right)+2 \epsilon\right) \leq \lim _{j \rightarrow \infty} C \epsilon^{-12} j^{-1 / 4}=0 .
$$

This holds for every $\epsilon>0$, and we have thus proved (3).

\section{ACKNowledgement}

The authors wish to thank the referee for pertinent comments, which helped to improve the paper. 


\section{REFERENCES}

1. A. Beurling and P. Malliavin, On the closure of characters and the zeros of entire functions, Acta Math. 118 (1967), 79-93. MR 35:654

2. G. Chystyakov and Yu. Lyubarskii, Random perturbations of Riesz bases from exponentials, Ann. Inst. Fourier (to appear).

3. P. Koosis, The Logarithmic Integral II, Cambridge University Press, Cambridge, 1992. MR 94i:30027

4. K. Seip and A. Ulanovskii, Random exponential frames, J. London. Math. Soc. 53 (1996), 560-568. CMP 96:14

Department of Mathematical Sciences, Norwegian University of Science and TechNOLOGY, N-7034 Trondheim, Norway

E-mail address: seip@math.unit.no

School of Science and Technology, Høgskolen i Stavanger, P. O. Box 2557, UllandhaUg, N-4004 Stavanger, Norway

E-mail address: alex-u@hauk.hsr.no 